\title{
mHealth: a narrative synthesis of evidence of its application in improving childhood immunization coverage
}

\author{
Surajudeen Abiola Abdulrahman ${ }^{1}$, Maarufat Olaide Olaosebikan ${ }^{2}$ \\ ${ }^{1}$ Department of Public Health Medicine, Penang Medical College, George Town, Penang, Malaysia; ${ }^{2}$ Department of Instructional Design and \\ Technology, Open University of Malaysia, Kuala Lumpur, Malaysia \\ Contributions: (I) Conception and design: SA Abdulrahman; (II) Administrative support: MO Olaosebikan; (III) Provision of study material or patients: \\ SA Abdulrahman; (IV) Collection and assembly of data: All authors; (V) Data analyses and interpretation: All authors; (VI) Manuscript writing: All \\ authors; (VII) Final approval of manuscript: All authors. \\ Correspondence to: Dr. Surajudeen Abiola Abdulrahman. Department of Public Health Medicine, Penang Medical College, 10450 George Town, \\ Penang, Malaysia. Email: abdulsuraj@gmail.com or surajudeen@pmc.edu.my.
}

\begin{abstract}
Under-5 mortality from preventable causes remains a significant problem in many parts of the developing world. Deaths from vaccine-preventable diseases (VPDs) continue to challenge the health care system of these countries, despite unrelenting efforts to expand routine immunization coverage. Despite the proven efficacy of these vaccines, several supply and demand-side factors, as well as other contextspecific factors have been associated with the poor uptake and completion rates, and the consequent high mortality rates from VPDs in these countries. In addition to specific policy and health system-related approaches to addressing these factors, inconsistent literature evidence suggests the potential efficacy of several range of interventions such as promoting antenatal care attendance and hospital delivery, providing education, financial incentives and deployment of mobile health technology (mHealth: SMS and telephone call reminders), in improving childhood immunization coverage in several countries across Africa, Asia and North America. This mini-review explored the diversity and quality of evidence regarding the application of mHealth in improving childhood immunization coverage. Following a brief search on Scimago Journal and Country Rank using a combination of relevant subject areas and subject categories, we finally evaluated 15 articles-mainly systematic reviews [3] and randomized controlled trials (RCTs) [12] — published in 12 top Q1 impact factor journals between 2010 and 2017. Overall, we found moderate to high-quality literature evidence suggesting the efficacy of mHealth interventions used singly or in combination with other interventions in improving childhood immunization coverage across several rural and urban settings around the world. While these evidences represent the larger consensus, we recommend that more attention should be given to detailed analysis of factors or circumstances that lead to negative results of these interventions in areas where such have been observed or reported.
\end{abstract}

Keywords: mHealth; childhood immunization coverage; vaccine-preventable diseases (VPDs); routine immunization; under-5 mortality

Received: 28 September 2017; Accepted: 23 October 2017; Published: 10 November 2017.

doi: 10.21037/jhmhp.2017.10.01

View this article at: http://dx.doi.org/10.21037/jhmhp.2017.10.01

\section{Introduction}

The unabating disproportional rate of morbidity and mortality from vaccine-preventable diseases (VPDs) among under-5 children in developing countries continues to generate discussions on the need for concerted efforts and innovative strategies to address factors militating against the achievement of universal access to immunization by year 2020, as initially conceptualized by the World Health Assembly's Global Vaccine Action Plan (GVAP). The GVAP is a global agenda signed in 2012 by 194 Member 


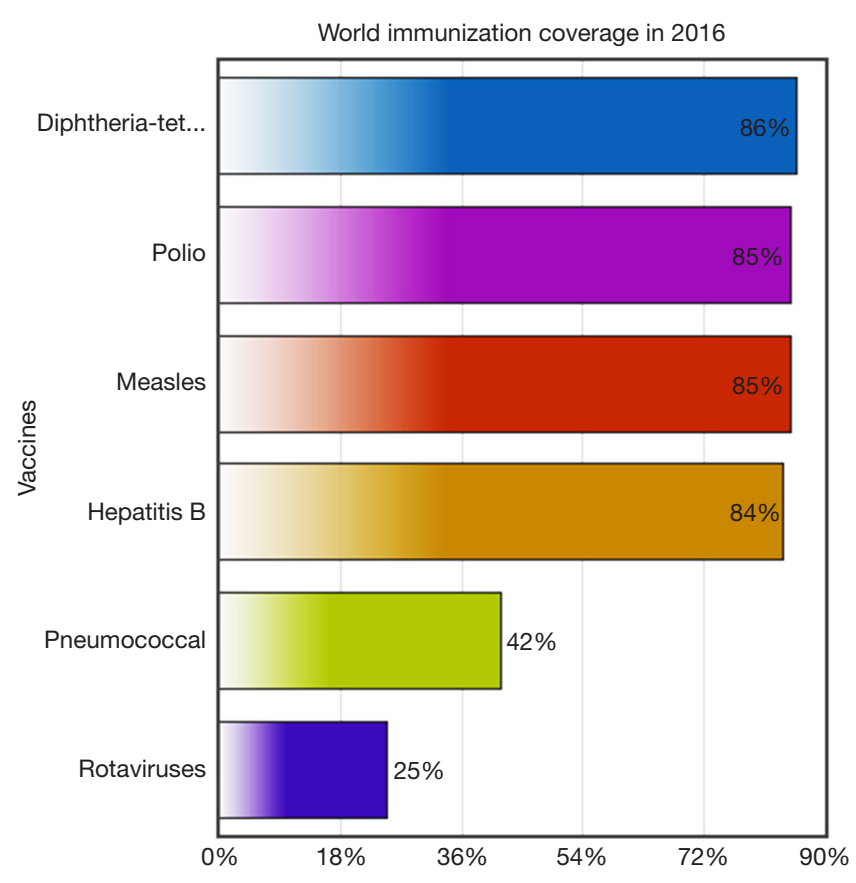

Figure 12016 global immunization coverage rates for specific vaccine types. Source: WHO, 2017.

States, which provides a "framework to prevent millions of deaths by 2020 through more equitable access to existing vaccines for people in all communities" (1). In recognition of the less than desired gains achieved through the GVAP and the need to address other challenges relating to lower than expected immunization coverage and slower adoption of newer vaccines by low- and middle-income countries, a renewed global commitment to end preventable deaths of newborns and children under 5 years of age by 2030, was agreed and documented in the Sustainable Development Goals (2).

There is no doubt that vaccines have helped drive the reduction in childhood mortality. According to a July 2017 report by World Health Organization (WHO), an estimated 2 to 3 million annual deaths from VPDs are saved by immunization (3). The potential to save additional 1.5 million annual deaths by reaching an estimated 19.5 million infants who currently miss out on basic immunization largely depends on our collective capacity and resolve to improve the Global vaccination coverage rate further from $86 \%$, on which it has stalled over the past few years. Efforts are required to address the gaps in coverage rates for the different immunizations (Figure 1), as well as the variations in coverage rates that exist across regions and countries (3).

Despite evidence suggesting efficacy of these vaccines in preventing childhood mortality, and the global adoption of Expanded Programme on Immunization (EPI), several supply and demand-side factors, as well as other contextspecific factors have been associated with the poor uptake and completion rates, and the consequent high mortality rates from VPDs, especially in developing countries. Factors on the supply side are believed to include inadequate funding, inefficient procurement, logistics and supply chain management system, poor governance, inefficient service delivery and poor data management (4). On the demand side, several socioeconomic factors such as maternal education, age, occupation, marital status, residence, antenatal attendance, family size, access to media, vaccine avoidance due to misconceptions about adverse events following immunization (AEFI), household wealth and place of delivery $(5-12)$ have been identified as potential barriers to immunization uptake and completion rates. Other factors include geographical location (ruralurban disparities in immunization completion rates), and difficulty in reaching health facilities (13). Other contextspecific factors that affect refusal of childhood vaccination include concerns about vaccine components (based mainly on religious reasons), low risk perception on severity of infectious diseases, and a trusting relationship with a natural healer or other respected person who doubts vaccination safety and effectiveness $(14,15)$. Studies have reported parents' refusal of vaccination on account of perception of an overload of the immune system caused by combination of vaccines (16), as well as the perception that vaccination is more risky than non-immunization (17).

Over the past decade, several policy and health systemrelated approaches to addressing these factors have been reported. Strategies that aimed to improve antenatal care attendance and hospital deliveries have been reported to improve immunization completion rates in several parts of the world (18-22). Additionally, interventions such as public education or knowledge translation (23), provision of financial incentives, and recently, the adoption of mobile health technology through provision of immunization reminders on mobile devices (SMS and telephone calls) and apps have demonstrated promising results in improving immunization coverage in Kenya, Guatemala, Zimbabwe, Pakistan, and China, albeit with a few inconsistencies (24-29). The aim of this review was to explore the diversity and quality of evidence regarding the application of mHealth in improving childhood immunization coverage. 


\section{Methods}

This was a scoping narrative review and synthesis of evidence from systematic reviews and randomized controlled trials (RCTs) suggesting efficacy or otherwise of several range of interventions (with a specific focus on mHealth) aimed at improving immunization coverageuptake and completion rates-as well as timeliness of receipt of scheduled immunizations. We conducted a brief search of Scimago Journal and Country Rank using a combination of subject areas and subject categories to identify top ranking Q1 impact factor journals in the respective categories. We used several search term combinations including 'Medicine' + 'Immunology and Allergy' AND 'Infectious Diseases' AND 'Public Health, Environmental and Occupational Health'. We searched for additional journals using a combination of 'Immunology and Microbiology' + 'Immunology and Microbiology (miscellaneous)'. We selected the top 20 open access journals in each category. We further examined these 80 journals to determine those that have published between 2010 and 2017, articles related to our subject area by using the terms 'Childhood' AND 'Immunization' OR 'Vaccination', 'Immunization' OR 'Vaccination' AND 'Coverage' 'Immunization' OR 'Vaccination' AND 'Uptake' 'Immunization' OR 'Vaccination' AND 'Completion rates' 'Immunization' OR 'Vaccination' AND 'Timeliness'. We also searched for terms such as 'financial incentives' AND/OR 'mHealth' AND/OR 'text messaging' OR 'SMS'. We further screened the 12 journals in our list at this stage, for articles that were either systematic reviews and/or RCTs and finally included three systematic reviews and 12 RCTs published in Q1 impact factor journals between 2010 and 2017. We retrieved the full text articles and read them in detail making special notes on the study population, intervention design and implementation, outcome measures, analysis and potentials for bias in the study design. Essentially, we applied a four-step process described by Popay et al. in this synthesis: (I) examined the theoretical basis of the included studies; (II) developed a preliminary synthesis; (III) explored relationships within and between studies; and (IV) assessed robustness of the synthesis.

\section{Results and discussion}

Evidence for and against efficacy of mHealth interventions in improving childhood immunization coverage

The ubiquitous availability of mobile phones (30) and devices suggest that the targeted application of mobile phone technologies to improving childhood immunization uptake and completion rates can be very successful in addressing demand side factors such as forgetting immunization appointments, not knowing the vaccine schedule, or incurring transportation costs to clinics/ hospitals, to improve immunization uptake.

In two RCTs conducted in United States of America, text-messaging was reported to improve influenza vaccination rates among low-income, urban, ambulatory children, adolescents and obstetric populations $(31,32)$. Similar results suggesting efficacy of text messaging $(26,33,34)$, a combination of text messaging and financial incentives (24), immunization camps and/or food incentives $(35,36)$ have been reported in both rural and urban settings of Zimbabwe, Kenya, Bangladesh, India and Pakistan.

Earlier studies conducted prior to the mHealth boom include a 2010 study conducted in India by Banerjee et al. (35) which provided evidence from a three-arm intervention study among 1,640 children aged 1-3 years from 134 villages, that while improving supply-side factors through regular and assured immunization services (village monthly immunization camps) yielded modest increase in immunization rates compared to controls, providing additional food incentives was more cost-effective and had larger positive impact on uptake of immunization services in resource-poor settings. Similar results were reported in an intervention study conducted in Karachi, Pakistan by Chandir et al. (36) in which Diphtheria-Tetanus-Pertussis (DTP) immunization completion rates was reported to be twice higher at 18 weeks of age among infants whose mothers received food coupon incentives compared to controls.

A 2012 intervention study conducted in the United States by Stockwell et al. (32) among 9,213 children and adolescents aged 6 months to 18 years was one of the earliest to provide reasonable evidence of the effect of textmessage reminders in improving influenza vaccination rates among low-income urban populations during the 2010-2011 influenza season. Subsequently, further concrete evidence of the efficacy of SMS-based interventions in improving immunization uptake, completion rates and timeliness in low-income urban to rural populations were provided by studies conducted in Zimbabwe, Kenya and Bangladesh by Bangure et al. (26), Haji et al. (33) and Uddin et al. (34). Bangure and colleagues demonstrated in an intervention study among 304 children conducted in Kadoma Zimbabwe that SMS reminders (in addition to 
routine health education) sent to mothers of the children not only increased 14-week immunization coverage by $20 \%$ among intervention (95\%) compared to control group ( $75 \%)$, but also improved timeliness of immunization receipt in the intervention compared to the control group respondents who received only routine health education. These findings were consistent with reports of a 2014 three-arm intervention study conducted in Kenya by Haji et al. (33) in which 1,116 children aged below 12 months were randomly assigned to receive SMS reminders or stickers provided to their parents to ensure they bring their children for second and third dose of pentavalent vaccine, or to the control group (routine reminder) who received paper-based appointment scheduling/reminder. Authors reported significant lower odds of drop-outs among the SMS group compared to the control group, while no differential efficacy was recorded for the sticker group. Evidence from a 2015 Bangladeshi quasi-experimental study conducted by Uddin et al. was very much similar. Authors had provided SMS immunization reminders to mothers of children aged $0-11$ months in the intervention group and recorded a $29.5 \%$ and $27.1 \%$ higher immunization coverage rates among intervention versus control participants in rural and urban areas, respectively.

Building on the established evidence of the efficacy of SMS in addressing demand-side factors affecting childhood immunization coverage, some researchers have explored the comparative effectiveness of SMS alone versus a combination of SMS and other incentives such as financial incentives, especially in populations with perceived low literacy rates, low to high background immunization rates and questionable acceptability of SMS among segments of the population. One of such novel studies is a 2017 high quality four-arm cluster randomized controlled trial (MSIMU) conducted in rural, western Kenya by Gibson and colleagues (24) in which 2,018 caregivers and their infants (infant-caregiver pairs) from 152 villages were randomized to the control, SMS only, SMS plus 75 Kenya Shillings (KES), and SMS plus 200 KES groups. The authors report a significantly higher likelihood of achieving full immunization coverage at 12 months of age by the SMS plus 200 KES group compared to controls. They found no significant differences in the full immunization coverage at 12 months in either the SMS only or SMS plus $75 \mathrm{KES}$ groups compared with the control arm.

Systematic reviews by Das et al. (37) and Odone et al. (38) echoed these findings on a broader scale. While Das and colleagues acknowledged the potential efficacy of interventions such as implementing vaccination requirement in school, sending reminders, and national permissive recommendation for adolescent vaccination in improving immunization uptake and leading to a decline in prevalence of HPV and genital warts, incidence of measles and pertussis, rubella susceptibility and varicella deaths; they recommended further stronger evidence of the efficacy of these interventions in low-and-middle income countries. Similarly, Odone and colleagues found some evidence that text messaging, accessing immunization campaign websites, using patient-held web-based portals and computerized reminders increased immunization coverage rates. They recommended further studies to enable concrete evaluation of the effectiveness of use of social networks, email communication and smartphone application interventions.

Meanwhile, Johri et al. (23) had demonstrated in another systematic review and meta-analysis of data from 11,512 participants enrolled in 11 studies that demandside interventions comprising essentially of education and knowledge translation studies and use of incentives were effective strategies in improving childhood vaccination coverage in low-and middle-income countries.

Conflicting evidence was reported by a study conducted by Chen and colleagues in rural China regarding effectiveness of a smartphone app in improving childhood immunization coverage. The authors reported no significant difference in full vaccination coverage rates recorded between village doctors who used EPI app and reminder text messages compared to village doctors in the control group who used their usual procedures and text messages (29). Similarly, Gibson et al., in the M-SIMU cluster RCT conducted in Kenya, reported that SMS alone did not improve immunization coverage at 12 months of age (24).

Notwithstanding the ethical concerns that arises with the use of financial incentives in driving demand for services (39), these evidences suggest that not only are mHealth applications towards improving childhood immunization coverage feasible and acceptable (25,40-42), they have also been demonstrated to have significant efficacy or effectiveness in addressing demand-side barriers to childhood immunization coverage in several parts of the world $(37,38)$. This is also consistent with findings of mHealth efficacy in driving behavior change associated with, but not limited to, medication adherence and outpatient clinic attendance for HIV (43) and other chronic disease care services.

This was largely a narrative synthesis of evidence in which we applied unconventional but standardized 
approaches in our search strategies and made no attempt to provide statistical estimates of overall efficacy of the interventions considered in this review. Given our approach to literature search, it is possible that we might have omitted some studies that provide useful information on the subject simply because they were not open access or published in top Q1 impact factor journals. We were more interested in demonstrating the chronology of evidence, diversity of results and perceived quality of the primary studies included in this review, while also using evidence from a scoping review of other systematic reviews to support our analysis. To this extent, we did not strictly apply the Cochrane collaboration criteria to assess risk of bias in the studies included in this review.

Although narrative reviews often take a less formal and rigorous approach compared to a systematic review and meta-analysis, nonetheless, they are considered valuable and important educational tool in continuing medical education (44), especially when a blended approach such as reporting methodology, search terms, databases used, and inclusion and exclusion criteria are provided (45), as was the case in this review.

\section{Conclusions}

Moderate to high quality evidence supports the overwhelming consensus on the effectiveness of mHealth interventions-used singly or in combination with other interventions-in improving childhood immunization uptake and completion rates across several regions of the world. Its potential to improve timeliness of immunization receipt has also been suggested. Arguments and evidences regarding inefficacy of mHealth in addressing demand-side barriers to immunization coverage are few and unpopular, and likely associated with contextual factors like background immunization coverage rates (overall and specific rates for different immunizations), content of the message, costs, and literacy level among others. Although mHealth interventions are generally perceived to be cost-effective, further studies are required to provide wider and more concrete evidence of its cost-effectiveness ratio compared to other types of interventions aimed at improving childhood immunization coverage.

\section{Acknowledgments}

Funding: None.

\section{Footnote}

Conflicts of Interest: Both authors have completed the ICMJE uniform disclosure form (available at http://dx.doi. org/10.21037/jhmhp.2017.10.01). The authors have no conflicts of interest to declare.

Ethical Statement: The authors are accountable for all aspects of the work in ensuring that questions related to the accuracy or integrity of any part of the work are appropriately investigated and resolved.

Open Access Statement: This is an Open Access article distributed in accordance with the Creative Commons Attribution-NonCommercial-NoDerivs 4.0 International License (CC BY-NC-ND 4.0), which permits the noncommercial replication and distribution of the article with the strict proviso that no changes or edits are made and the original work is properly cited (including links to both the formal publication through the relevant DOI and the license). See: https://creativecommons.org/licenses/by-nc-nd/4.0/.

\section{References}

1. Global Vaccine Action Plan 2011-2020. Available online: http://www.who.int/immunization/global_vaccine_ action_plan/GVAP_doc_2011_2020/en/ (Accessed 10 September, 2017).

2. Transforming our world: The 2030 agenda for sustainable development. Available online: https://sustainabledevelopment.un.org/content/ documents/21252030\%20Agenda $\% 20$ for $\% 20$ Sustainable\%20Development\%20web.pdf (Accessed 10 September, 2017).

3. World Health Organization. Immunization Coverage Fact Sheet. Available online: http://www.who.int/mediacentre/ factsheets/fs378/en/ (Accessed 10 September, 2017).

4. Ophori EA, Tula MY, Azih AV, et al. Current Trends of Immunization in Nigeria: Prospect and Challenges. Trop Med Health 2014;42:67-75.

5. Tagbo BN, Eke CB, Omolowo BI, et al. Vaccination Coverage and its Determinants in Children Aged 11-23 Months in an Urban District of Nigeria. World Journal of Vaccines 2014;4:175-83.

6. Elizabeth K, George K, Raphael N, et al. Factors Influencing Low Immunization Coverage Among Children Between 12 - 23 Months in East Pokot, Baringo Country, Kenya. Int J Vaccines Vaccin 2015;1:00012. 
7. Arooj S, Ali S, Baber N, et al. Socioeconomic factors effecting polio vaccination in Pakistan. Health 2013;5:892-7.

8. Amin R, De Oliveira TJ, Da Cunha M, et al. Factors limiting immunization coverage in urban Dili, TimorLeste. Glob Health Sci Pract 2013;1:417-27.

9. Bbaale E. Factors Influencing Childhood Immunization in Uganda. J Health Popul Nutr 2013;31:118-29.

10. Oliveira MF, Martinez EZ, Rocha JS. Factors associated with vaccination coverage in children $<5$ years in Angola. Rev Saude Publica 2014;48:906-15.

11. Lakew Y, Bekele A, Biadgilign S. Factors influencing full immunization coverage among 12-23 months of age children in Ethiopia: evidence from the national demographic and health survey in 2011. BMC Public Health 2015;15:728.

12. Vonasek BJ, Bajunirwe F, Jacobson LE, et al. Do Maternal Knowledge and Attitudes towards Childhood Immunizations in Rural Uganda Correlate with Complete Childhood Vaccination? PLoS One 2016;11:e0150131.

13. Adedokun ST, Uthman OA, Adekanmbi VT, et al. Incomplete childhood immunization in Nigeria: a multilevel analysis of individual and contextual factors. BMC Public Health 2017;17:236.

14. Gust D, Brown C, Sheedy K, et al. Immunization attitudes and beliefs among parents: beyond a dichotomous perspective. Am J Health Behav 2005;29:81-92.

15. Benin AL, Wisler-Scher DJ, Colson E, et al. Qualitative analysis of mothers' decision-making about vaccines for infants: the importance of trust. Pediatrics 2006;117:1532-41.

16. Hilton S, Petticrew M, Hunt K. Combined vaccines are like a sudden onslaught to the body's immune system: parental conerns about vaccine 'overload' and 'immunevulnerability'. Vaccine. 2006;24:4321-7.

17. Smailbegovic MS, Laing GJ, Bedford H. Why do parents decide against immunization? The effect of health beliefs and health professionals. Child Care Health Dev 2003;29:303-11.

18. Phathammavong O, Ali M, Souksavat S, et al. Antenatal care among ethnic populations in Louang Namtha Province, Lao PDR. Southeast Asian J Trop Med Public Health 2010;41:705-16.

19. Etana B, Deressa W. Factors associated with complete immunization coverage in children age 12-23 months in Ambo Woreda, Central Ethiopia. BMC Public Health 2012;12:566.

20. Sullivan MC, Tegegn A, Tessema F, et al. Minding
Immunization Gap: Family Characteristics Associated with Completion Rates in Rural Ethiopia. J Community Health 2010;35:53-9.

21. Shrivastwa N, Gillespie BW, Kolenic GE, et al. Predictors of Vaccination in India for Children Aged 12-36 Months. Am J Prev Med 2015;49:S435-44.

22. Nankabirwa V, Tylleskär T, Tumwine JK, et al. Maternal education is associated with vaccination status of infants less than 6 months in Eastern Uganda: a cohort study. BMC Pediatr 2010;10:92.

23. Johri M, Pérez MC, Arsenault C, et al. Strategies to increase the demand for childhood vaccination in low-and middle-income countries: a systematic review and metaanalysis. Bull World Health Organ 2015;93:339-346C.

24. Gibson DG, Ochieng B, Kagucia EW, et al. Mobile phone-delivered reminders and incentives to improve childhood immunisation coverage and timeliness in Kenya (M-SIMU): a cluster randomised controlled trial. Lancet Glob Health 2017;5:e428-e438.

25. Domek GJ, Contreras-Roldan IL, O'Leary ST, et al. SMS text message reminders to improve infant vaccination coverage in Guatemala: A pilot randomized controlled trial. Vaccine 2016;34:2437-43.

26. Bangure D, Chirundu D, Gombe N, et al. Effectiveness of short message services reminder on childhood immunization programme in Kadoma, Zimbabwe - a randomized controlled trial, 2013. BMC Public Health 2015;15:137.

27. Kazi AM, Murtaza A, Kazi AN, et al. Effect of mobile phone text messages reminders on uptake of routine immunization among children in Pakistan-study protocol for a randomized clinical trial. J Vaccines Vaccin 2016;7:333.

28. Kazi AM. The role of mobile phone-based interventions to improve routine childhood immunisation coverage. Lancet Glob Health 2017;5:e377-e378.

29. Chen L, Du X, Zhang L, et al. Effectiveness of a smartphone app on improving immunization of children in rural Sichuan Province, China: a cluster randomized controlled trial. BMC Public Health 2016;16:909

30. International Telecommunications Union. The World in 2015: ICT Facts and Figures. Available online: http://www. itu.int/en/ITU-D/Statistics/Documents/facts (Accessed 10 September, 2017).

31. Moniz MH, Hasley S, Meyn LA, et al. Improving influenza vaccination rates in pregnancy through text messaging: a randomized controlled trial. Obstet Gynecol 2013;121:734-40. 
32. Stockwell MS, Kharbanda EO, Martinez RA, et al. Effect of a text messaging intervention on influenza vaccination in an urban, low-income pediatric and adolescent population: a randomized controlled trial. JAMA 2012;307:1702-8.

33. Haji A, Lowther S, Ngan'ga, Z, et al. Reducing routine vaccination dropout rates: evaluating two interventions in three Kenyan districts, 2014. BMC Public Health 2016;16:152.

34. Uddin MJ, Shamsuzzaman M, Horng L, et al. Use of mobile phones for improving vaccination coverage among children living in rural hard-to-reach areas and urban streets of Bangladesh. Vaccine 2016;34:276-83.

35. Banerjee AV, Duflo E, Glennerster R, et al. Improving immunisation coverage in rural India: clustered randomised controlled evaluation of immunisation campaigns with and without incentives. BMJ 2010;340:c2220.

36. Chandir S, Khan AJ, Hussain H, et al. Effect of food coupon incentives on timely completion of DTP immunization series in children from a low-income area in Karachi, Pakistan: a longitudinal intervention study. Vaccine 2010;28:3473-8.

37. Das JK, Salam RA, Arshad A, et al. Systematic Review and Meta-Analysis of Interventions to Improve Access and Coverage of Adolescent Immunizations. J Adolesc Health 2016;59:S40-S48.

38. Odone A, Ferrari A, Spagnoli F, et al. Effectiveness of interventions that apply new media to improve vaccine

doi: 10.21037/jhmhp.2017.10.01

Cite this article as: Abdulrahman SA, Olaosebikan MO. mHealth: a narrative synthesis of evidence of its application in improving childhood immunization coverage. J Hosp Manag Health Policy 2017;1:6. uptake and vaccine coverage: a systematic review. Hum Vaccin Immunother 2015;11:72-82.

39. Lunze K, Paasche-Orlow MK. Financial incentives for healthy behavior: ethical safeguards for behavioral economics. Am J Prev Med 2013;44:659-65.

40. Rossing E, Ravn H, Batista CS, et al. MHealth to Improve Measles Immunization in Guinea-Bissau: Study Protocol for a Randomized Controlled Trial. JMIR Res Protoc 2016;5:e158.

41. Wakadha H, Chandir S, Were EV, et al. The feasibility of using mobile-phone based SMS reminders and conditional cash transfers to improve timely immunization in rural Kenya. Vaccine 2013;31:987-93.

42. Sadoh AE, Okungbowa E. Nigerian mothers opinion of reminder/recall for immunization. Niger J Paediatr 2014;41:38-42.

43. Abdulrahman SA, Rampal L, Ibrahim F, et al. Mobile phone reminders and peer counseling improve adherence and treatment outcomes of patients on ART in Malaysia: A randomized clinical trial. PLoS One 2017;12:e0177698.

44. Bernardo WM, Nobre MR, Jatene FB. Evidence based clinical practice. Part II - searching evidence databases. Rev Assoc Med Bras 2004;50:104-8.

45. Popay J, Roberts H, Sowden A, et al. Guidance on the conduct of narrative synthesis in systematic reviews. A product from the ESRC methods programme. Version 2006; 1:b92. 